\title{
The disturbance signal detection method of power quality based on MEEEMD
}

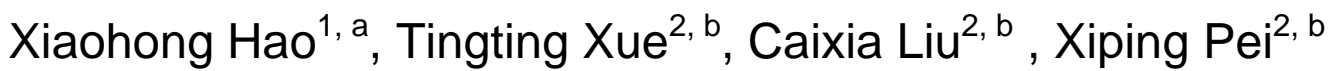 \\ ${ }^{1}$ College of Computer and Communication, Lanzhou University of Technology, Lanzhou 730050, \\ China; \\ ${ }^{2}$ College of Electrical and information Engineering, Lanzhou University of Technology, Lanzhou \\ 730050, China \\ aemail:whscdx1126@163.com, bemail:836706871@qq.com
}

Key words: mode mixing; MEEMD; Hilbert transform; power quality

\begin{abstract}
To solve the problem adding white noise cannot be completely neutralized and it is not complete of Ensemble Empirical Mode Decomposition(MEEMD), the paper propose that combine improvement of EEMD and Hilbert transform to detect power quality disturbance. First ,decompose power quality disturbance signals and get intrinsic mode functions(IMF) components. Second ,the IMFs after Hilbert transform to obtain accurate instantaneous characteristic at different times. The simulation results show that the MEEMD method has a clear effect to suppress mode mixing and it also applies to the mixed power quality detection.
\end{abstract}

\section{Introduction}

Now electricity is essential for people in life,but the power quality produces a series of problems due to the extensive use of shock, nonlinear loads in real life,such as unstable voltage, waveform distortion[1].In order to improve power quality,to ensure the safety and reliability of power supply and distribution,power quality disturbance detection has become a research focus.There are a large amount of literature using mathematical theories,such as short-time Fourier transform[2],S transform[3],wavelet transform method[4-6],Hilbert-Huang transform[7], mathematical morphology[8]and method of combining several algorithms to detect disturbances, as well as made a lot of achievements.Chinese-American Huang etc proposed Empirical Mode Decomposition(EMD) at the end of last century, it is an adaptive signal processing method of data,nonetheless, one of the major shortcomings occurring in the EMD is the mode mixing problem.Ensemble Empirical Mode Decomposition(EEMD) proposed by $\mathrm{Wu}$ when he add the white noise into the analyzed signal,which effectively reduces the mode mixing problem.But the drawback of EEMD are large amount of caclculation and iteration limit,its components corresponding to the IMF are not fully.Thus, Modified EEMD(MEEMD) with Hilbert method is proposed to detect and analyse transient power quality and mixed power quality disturbances.The simulation results show that MEEMD method not only supplement the deficiency of EEMD,but also has a inhibiting effect on the mode mixing problem,and can draw the instantaneous frequency and instantaneous amplitude and other information to location the disturbance sudden change time and quantitative testing.

\section{Modified EEMD(MEEMD) method}

\section{EMD and EEMD}

The EMD method is capable of decomposing a signal into a series of Intrinsic Mode Function(IMF), which has its own characteristics.An IMF is a function that satisfies the two following conditions:(1) in the whole data set,the number of extreme and the number of zero-crossing must either equal or differ at most by one;(2) at any point,the mean value of the envelope defined by the local maxima and the envelope defined by the local minima is zero.EEMD is proposed to prevent the mode mixing problem and obtain the IMF.The literature[6] is to add 
random white noise into the analyzed signal,the decomposition steps are as follows:

(1) Add different white noise to the analyzed signal;

(2) Decomposing analyzed signal of EMD;

(3) Cycle the steps(1) (2);

(4) Calculate the overall average of above results, to minimize the effect of the Gaussian white noise on the IMF, then obtain the final decomposition results.

\section{The MEEMD Method}

According to the research of Wu and Yeh,this paper proposed a Modified Ensemble Empirical Mode Decomposition (MEEMD).Detection of high frequency or intermittent signal is the biggest advantage of MEEMD.Assuming a non-stationary signal $X(\mathrm{t})$, MEEMD steps are as follows[7]:

(1) Zero mean white noise signal $n_{i}(\mathrm{t})$ and $-n_{i}(\mathrm{t})$ were added to $X(\mathrm{t})$ :

$$
\begin{aligned}
& X_{i}^{+}(\mathrm{t})=X(\mathrm{t})+a_{i} n_{i}(\mathrm{t}) \\
& X_{i}^{-}(\mathrm{t})=X(\mathrm{t})-a_{i} n_{i}(\mathrm{t})
\end{aligned}
$$

Where $n_{i}(\mathrm{t})$ and $a_{i}$ are white noise signal and the amplitude of white noise signal,respectively.i $=1,2, \cdots \cdots, N e, N e$ is the number of added white noise.

The EMD method decomposed $X_{i}^{+}(\mathrm{t})$ and $X_{i}^{-}(\mathrm{t})$ respectively to obtain the first order IMF $\left\{I_{i 1}^{+}(\mathrm{t})\right\}$ and $\left\{I_{i 1}^{-}(\mathrm{t})\right\} \quad(\mathrm{i}=1,2, \cdots, N e)$.Integrated average component obtained above: $I_{1}(\mathrm{t})=\frac{1}{2 N} \sum_{i=1}^{N e}\left[I_{i 1}^{+}(\mathrm{t})+I_{i 1}^{-}(\mathrm{t})\right]$

(2) If $I_{1}(\mathrm{t})$ is abnormal signal ,then return to step(1), until the IMF $I_{p}(\mathrm{t})$ to normal signal.

(3) Former $p-1$ component from decomposition of signal $X(\mathrm{t})$ :

$$
r(\mathrm{t})=X(\mathrm{t})-\sum_{j=1}^{p-1} I_{j}(\mathrm{t})
$$

(4) Finally,decomposing the residual signal $\mathrm{r}(\mathrm{t})$ by EMD method,then all IMFs represent different frequency components from high frequency to low frequency.

\section{Hilbert Transform}

The random signal $X(\mathrm{t})$, the Hilbert transform $Y(\mathrm{t})$ is defined as[7]:

$$
\mathrm{Y}(\mathrm{t})=\frac{1}{\pi} P \int_{-\infty}^{+\infty} \frac{X\left(\mathrm{t}^{\prime}\right)}{t-t^{\prime}} d t^{\prime}
$$

in which $P$ indicates the Cauchy principal value. Therefore, the analytic signal $\mathrm{Z}(\mathrm{t})$ is defined as:

$$
\begin{aligned}
& \mathrm{Z}(\mathrm{t})=X(\mathrm{t})+j Y(\mathrm{t})=\mathrm{a}(\mathrm{t}) \mathrm{e}^{j \theta(\mathrm{t})} \\
& \left\{\begin{array}{l}
a(\mathrm{t})=\sqrt{X^{2}(\mathrm{t})+Y^{2}(\mathrm{t})} \\
\theta(\mathrm{t})=\arctan \frac{X(\mathrm{t})}{Y(\mathrm{t})}
\end{array}\right.
\end{aligned}
$$

the instantaneous frequency as formula (7):

$$
\mathrm{f}(\mathrm{t})=\frac{1}{2 \pi} \frac{\mathrm{d} \theta(\mathrm{t})}{\mathrm{dt}}
$$

\section{The Hilbert detection method of power quality disturbance based on MEEMD}

In this paper,using Hilbert transform method of MEEMD to decompose disturbance signal into single componment signal.The processing steps are showed as follow:

(1) Decomposing original signal of MEEMD,get each order of IMF;

(2) Reasonable reconstruction of IMF,so that the decomposed components can be fully utilized;

(3) Calculate the corresponding a(t) and $\mathrm{f}(\mathrm{t})$ by Hilbert transform;

(4) Used to obtain maximum value to locate the start and end time;

(5) Obtain the amplitude and frequency of the disturbance by the mean fitting etc. 


\section{Simulation of power quality disturbance signal}

\section{The disturbance signal mathematical model}

In this paper,the simulation and verification of the common transient power quality and several mixed disturbance signal.The disturbance signal is generated in accordance with the method in reference[7],amplitude are used per unit,single disturbance signal(without noise) as shown in Table1.

Table1 Mode of power quality disturbance

\begin{tabular}{|l|c|}
\hline Disturbance type & \multicolumn{1}{|c|}{ Disturbance mode } \\
\hline Voltage dips & $f(\mathrm{t})=\left[1-\alpha\left(\varepsilon\left(\mathrm{t}_{1}\right)-\varepsilon\left(\mathrm{t}_{2}\right)\right)\right] \sin \omega_{0} t, \alpha=0.1 \sim 0.9,0.5 T<\mathrm{t}_{2}-\mathrm{t}_{1}<0.3 s$ \\
\hline Voltage swell & $f(\mathrm{t})=\left[1+\alpha\left(\varepsilon\left(\mathrm{t}_{1}\right)-\varepsilon\left(\mathrm{t}_{2}\right)\right)\right] \sin \omega_{0} t, \alpha=0.1 \sim 0.9,0.5 T<\mathrm{t}_{2}-\mathrm{t}_{1}<0.3 s$ \\
\hline Voltage interruption & $f(\mathrm{t})=\left[1-\alpha\left(\varepsilon\left(\mathrm{t}_{1}\right)-\varepsilon\left(\mathrm{t}_{2}\right)\right)\right] \sin \omega_{0} t, \alpha=0.9 \sim 1.0,0.5 T<\mathrm{t}_{2}-\mathrm{t}_{1}<0.3 s$ \\
\hline Pulse transient & $f(\mathrm{t})=\sin \omega_{0} t+\alpha\left(\varepsilon\left(\mathrm{t}-\mathrm{t}_{1}\right)-\varepsilon\left(\mathrm{t}-\mathrm{t}_{2}\right)\right), \alpha=0 \sim 2,1 \mathrm{~ms}<t_{2}-t_{1}<3 m s$ \\
\hline Transient & $f(\mathrm{t})=\sin \omega_{0} t+a e^{-b\left(\mathrm{t}-\mathrm{t}_{1}\right)} \sin \left(\mathrm{c} \omega_{0} t\right)\left(\varepsilon\left(\mathrm{t}_{1}\right)-\varepsilon\left(\mathrm{t}_{2}\right)\right), \mathrm{a}=0.1 \sim 0.8$, \\
oscillatory & $\mathrm{c}=10 \sim 40,0<t_{2}-t_{1}<2 T$ \\
\hline harmonic , inter- & $f(\mathrm{t})=\sin \omega_{0} t+\sum_{i=2}^{n} a_{i} \sin \left(\mathrm{m}_{i} \omega_{0} \mathrm{t}\right) a_{i}, \mathrm{~m}_{i}$ are the amplitude and frequence of the different \\
harmonic & frequence harmonics or inter- harmonic \\
\hline
\end{tabular}

\section{Analysis of power quality disturbance signal}

Simulate on the MATLAB plat, $3200 \mathrm{~Hz}$ as sampling frequency, disturbing signal parameters are as follows: Voltage sags, $\alpha=0.3, \mathrm{t}_{1}=0.13 \mathrm{~s}, \mathrm{t}_{2}=0.20 \mathrm{~s}$; Voltage swell, $\alpha=0.35, \mathrm{t}_{1}=0.15 \mathrm{~s}, \mathrm{t}_{2}=0.24 \mathrm{~s}$;Voltage interruption, $\alpha=0.95, \mathrm{t}_{1}=0.17 \mathrm{~s}, \mathrm{t}_{2}=0.19 \mathrm{~s}$; Transient pulse, $\alpha=1.7, \mathrm{t}_{1}=0.028 \mathrm{~s}, \mathrm{t}_{2}=0.03 \mathrm{~s}$; Transient oscillation's parameters: Oscillation amplitude, $a=0.6$, Relative coefficient, $c=15$, Attenuation coefficients, $b=10$, $\mathrm{t}_{1}=0.150 \mathrm{~s}, \mathrm{t}_{2}=0.180 \mathrm{~s}, \mathrm{t}_{1}, \mathrm{t}_{2}$ are the moments which is starting and ending of disturbance.

Fig. 1 is the voltage sags. Fig. 2 is the voltage swells and Figure 3 is the voltage interruption(amplitude is per unit), the figures show that it satisfy the IMF's conditions. Because of this, Hilbert change can be used directly to calculate the instantaneous of frequency and amplitude.

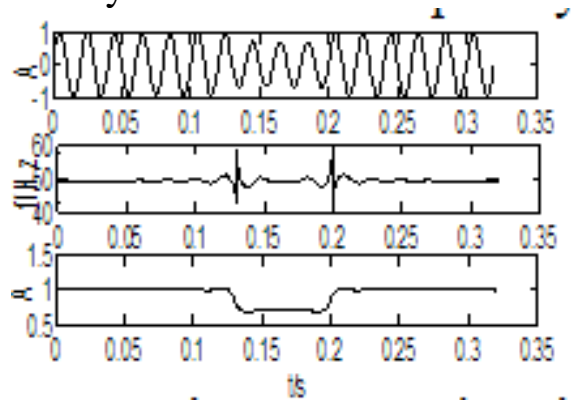

Fig. 1. Voltage sags signal

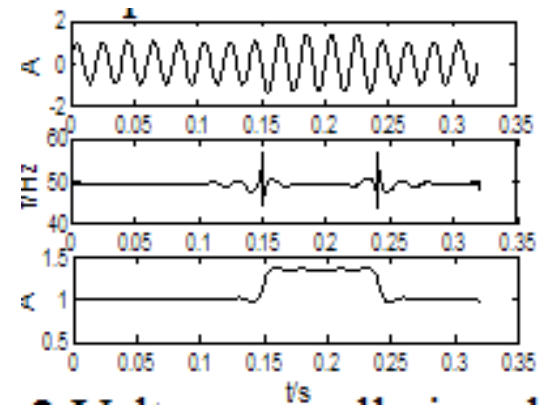

Fig.2 Voltage swell signal 


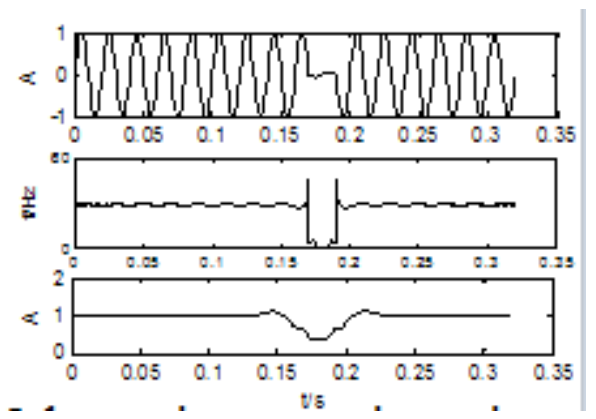

Fig.3 Voltage interruption signal

The figure show that 3 types of disturbance signal's amplitude has a volatility of different degree , the other time is the fundamental frequency $(50 \mathrm{~Hz})$ and is continuous except the Jump Point have some changes.

Transient pulse and transient oscillations are analyzed and simulated in the method of EEMD and MEEMD to get the IMF components.

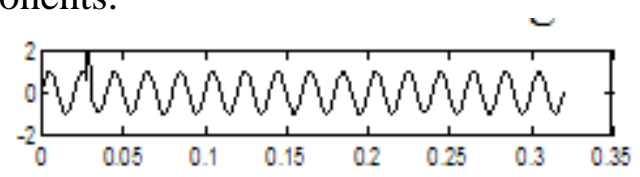

(a) Transient pulse signal

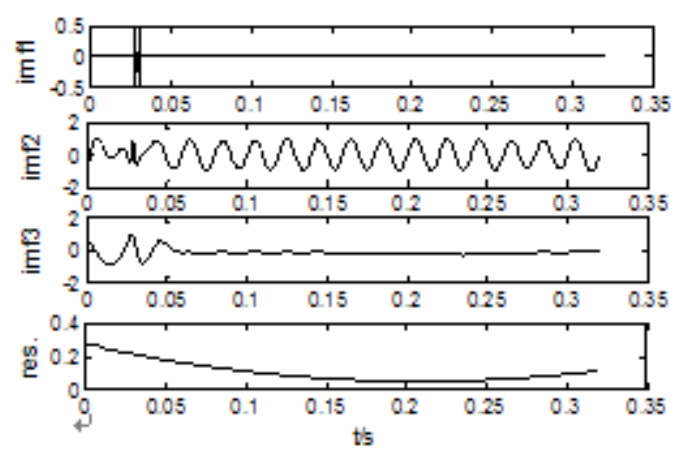

(b) EEMD decomposition

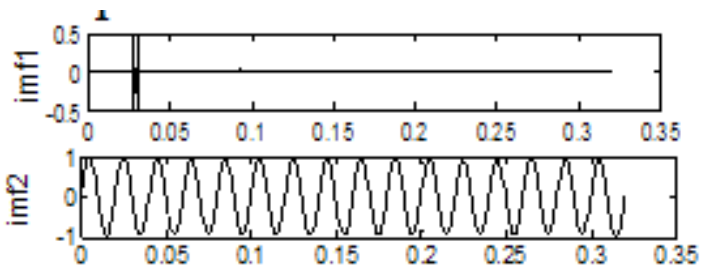

(c) MEEMD decomposition
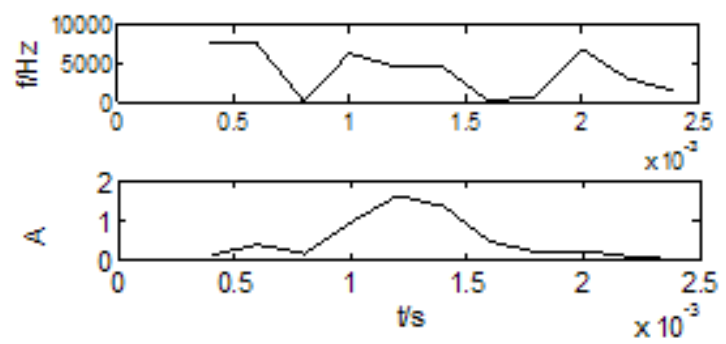

(d) Instantaneous frequency and amplitude of Hilbert

Fig.4. Analysis of transient pulse signal

The essence of transient disturbance is another model signal superpose on the original ideal signal. It can been seen from(b),(c) of figure 4, the EEMD decomposition can suppress EMD mode mixing by adding white noise;the MEEMD decomposition have no false components and the residual component is zero. 


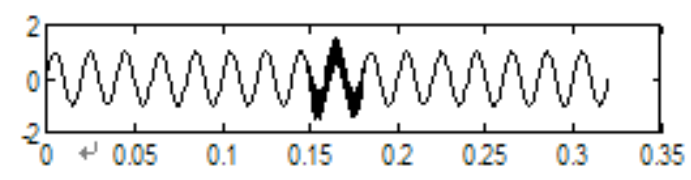

(a)Transient pulse signal
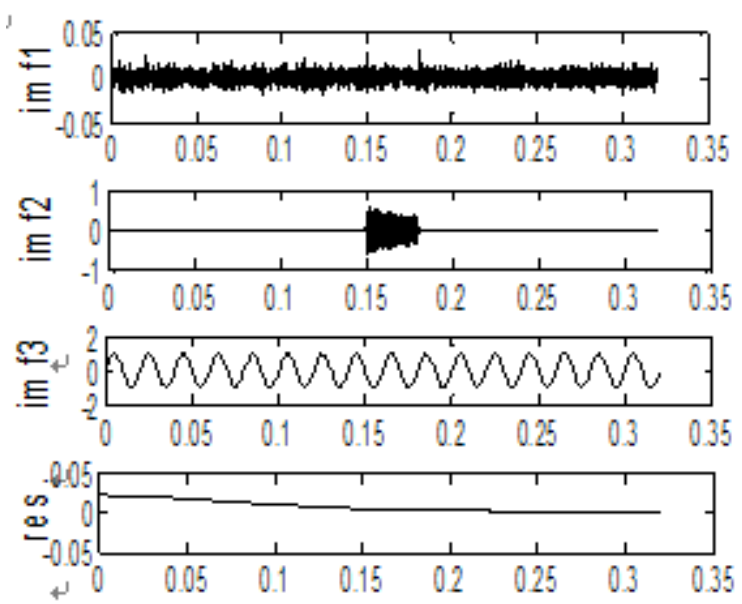

(b) EEMD decomposition

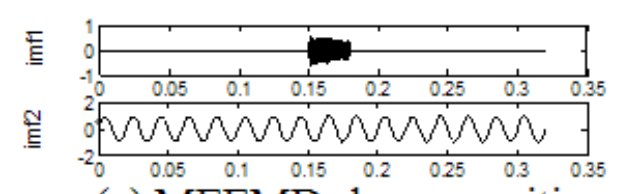

(c) MEEMD decomposition

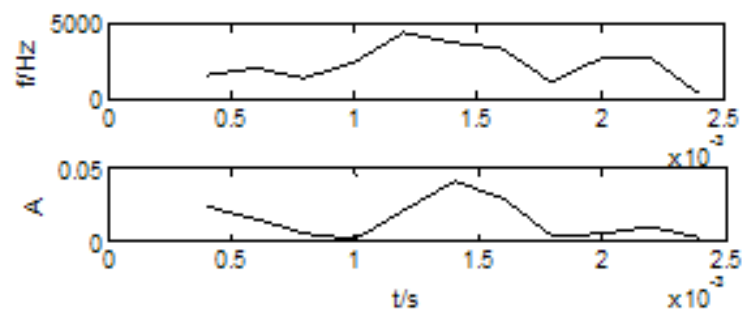

(d) Instantaneous frequency and amplitude of Hilbert

Fig.5. Analysis of oscillation transient signals

It can been seen from (b) (c) of Figure 5, the effect of decomposition is the same as Figure 4,it satisfy the IMF conditions, take Hilbert transform on IMF components which have clear physical meaning, as Figure 4(d), Figure 5(d). It can been know the components of disturbance start-end time, amplitude of disturbance and frequency after decompose the IMF.

\section{Analysis of combined disturbance}

In the paper the following three kinds of mixed disturbances are detected for analysis: voltage sags and harmonic, voltage swells and harmonic, transient oscillation and inter-harmonic.The IMF components after MEEMD decomposed as follow Figure 6-8.

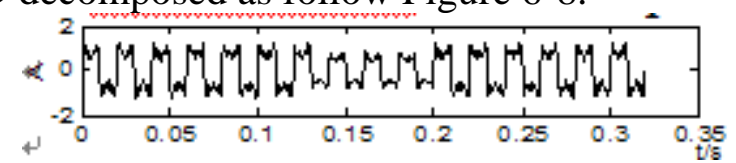

(a) voltage sags and harmonic disturbance

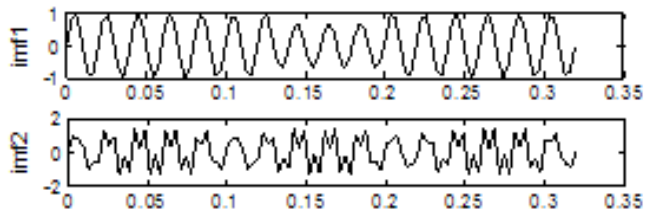

(b) IMF components after MEEMD 

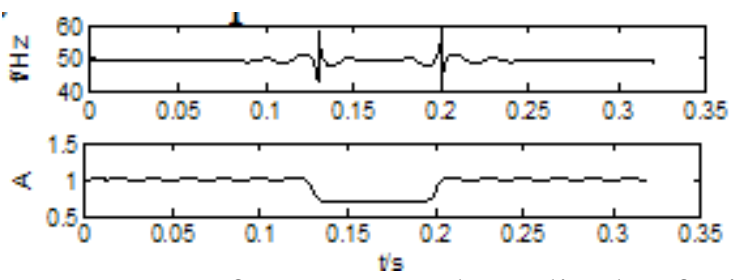

(c)Instantaneous frequency and amplitude of Hilbert

Fig.6.The MEEMD of voltage sags and harmonic disturbance

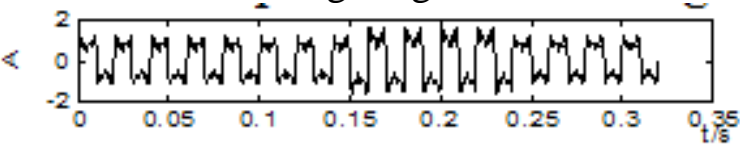

(a)voltage swells andharmonic disturbance

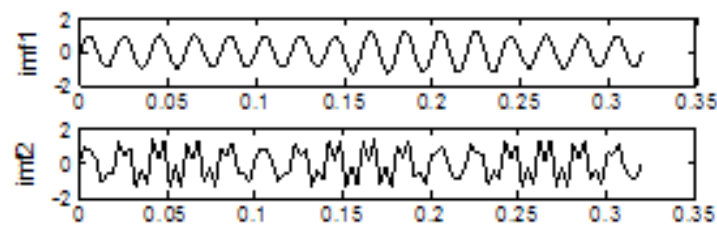

(b)IMF components after MEEMD
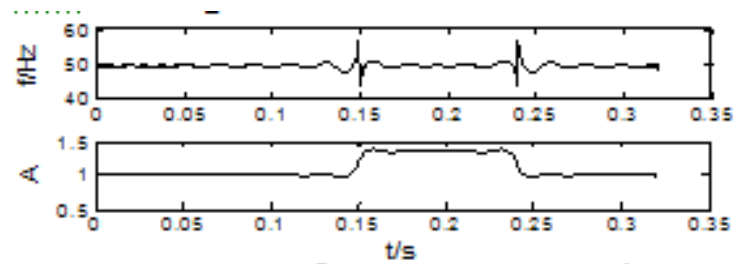

(c)Instantaneous frequency and amplitude of Hilbert

Fig.7 The MEEMD of voltage swells and harmonic disturbance

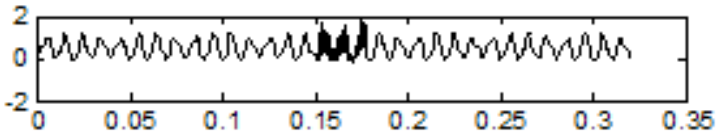

(a)transient oscillation and inter-harmonic disturbance

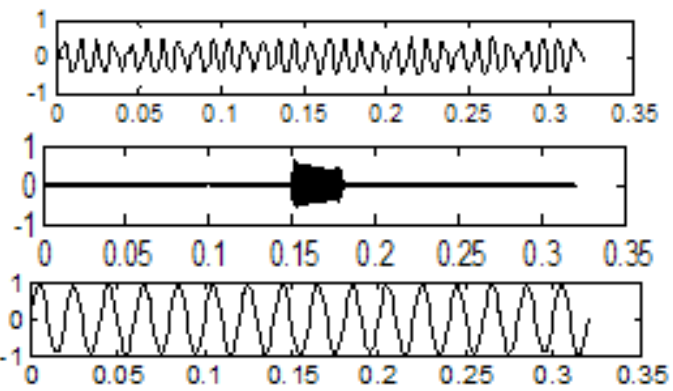

(b)IMF components after MEEMD
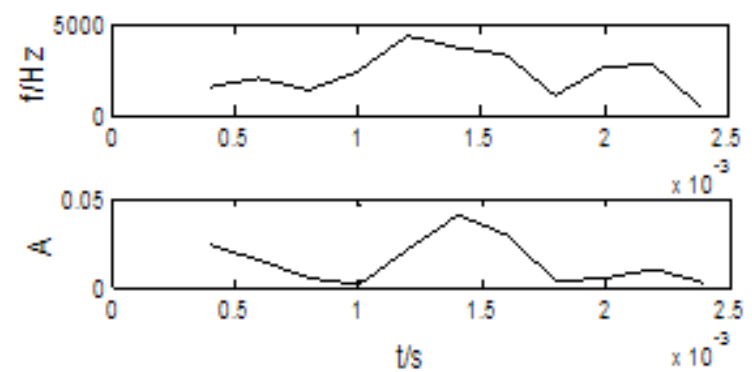

(c)Instantaneous frequency and amplitude of Hilbert

Fig. 8 The MEEMD of transient oscillation and inter-harmonic disturbance

Fig.6-8 show that MEEMD separate single disturbance accurately from mix power quality 
disturbance signal and decomposition of residual signal is approximately zero.Then Hilbert transform the IMF components which have clear physical meaning, obtain instantaneous frequency and amplitude curve and achieve the purpose of mixed power quality detection.

\section{Conclusion}

The EEMD method cannot improve the mode mixing problem of EMD,thus,the paper proposes a modified EEMD(MEEMD) method.The simulation of power quality disturbance signal show that MEEMD not only solve the mode mixing problem and get IMF of obvious physical meaning,but also meet the definition.By combining the Hilbert transform,it can accurately detect the mutation point of disturbance signal,and the amplitude and frequency parameters of disturbance signal.

\section{Acknowledgement}

In this paper, the research was sponsored by the National Natural Science Foundation of China(Project No. 51467009) and the Nature Science Foundation of Gansu Province (Project No.1310RJYA079).

\section{References}

[1] Huan Huang, Jiekang Wu. A Method to Locate Power Quality Disturbing Signal Based on Empirical Mode Decomposition[J]. Power System Technology,2010,34(5):41-45.

[2] Fengzhan Zhao, Rengang Yang. Voltage Sag Disturbance Detection Based on Short Time Fourier Transform[J]. Proceedings of the CSEE,2007,27(10):28-34,109.

[3] Fangwei Xu, Honggeng Yang, Maoqing Ye. Classification for Power Quality Short Duration Disturbances Based on Generalized S-transform[J]. Proceedings of the CSEE,2012,32(4):77-84.

[4] Jun He. Estimate of Wavelet Transform Based Power Quality and MATLAB Simulation[J].Sichuan Vocational and Technical College,2013,23(1):142-145.

[5] Tongyu $\mathrm{Xu}$, Zailin Piao, Chunling Chen. Application of lifting wavelet in transient power quality disturbance detection and location[J]. Transactions of the CSAE,2011,27(5):282-286.

[6] Huang $\mathrm{N}$ E,Wu Z.A review on Hilbert - Huang transform:Method and its applications to geophysical studies,Advances in Adaptive Data Analysis 2009,1:1-23.

[7] Huang N E,Shen Z,Long S R,et al.The empirical mode decomposition and the Hilbert spectrum for nonlinear and non-stationary time series,Proc.Roy.Soc.London,1998,454:903-995.

[8] Ke Hui, Gu Jie. Based on binary system wavelet and binary-coded decimal power quality signals detection and classification[J].Electric power automation equipment, 2009,29(7):67-71. 